\section{Satellite-sensed turbulent ocean structure}

RECENTLY Gower et al. ${ }^{1}$ have reported interesting results concerning the concentration of phytoplankton as measured by the Landsat multispectral scanner on 19 June 1976 in a region south of Iceland. The observed concentration fluctuation spectra is found to follow approximately a $k^{-2.92}$ law, close to the $k^{-3}$ law proposed by $\mathrm{Charney}^{2}$ for the energy spectrum of quasi-geostrophic flows. They conclude that this result is consistent with the hypothesis that phytoplankton behaves like a passive scalar convected by ocean currents.

The quasi-geostrophic theory of Charney ${ }^{2}$ predicts $k^{-3}$ laws for both horizontal kinetic energy and temperature variance, however, temperature is not a passive scalar, but the vertical derivative of the horizontal stream function. It is the constraints of total (kinetic plus available potential) energy conservation and potential enstrophy conservation

$$
\begin{aligned}
\frac{\mathrm{d}}{\mathrm{d} t}\left\langle\psi_{x}^{2}+\psi_{y}^{2}+\psi_{z}^{2}\right\rangle & =0 \\
\frac{\mathrm{d}}{\mathrm{d} t}\left\langle\psi_{x x}^{2}+\psi_{y y}^{2}+\psi_{z z}^{2}\right\rangle & =0
\end{aligned}
$$

together with the assumption of statistical isotropy, which yields for any of the derivatives $\psi_{x}, \psi_{y}, \psi_{z}$ a power spectrum following a $k^{-3}$ law in the potential enstrophy cascading range.

Now, if we consider a passive scalar convected by the quasi-geostrophic motion, as we may expect phytoplankton is, the situation must be quite different. For simplicity we neglect baroclinic effects: for pure two-dimensional turbulence, it can be shown ${ }^{3}$ using either phenomenological arguments or statistical closures, that the spectrum of a passive scalar is proportional to the spectrum of the quantity which cascades. Therefore in the enstrophy cascading inertial range, the spectrum of a passive scalar should follow a $k^{-1}$ law, like the enstrophy (and not the energy) spectrum. There seems to be no reason why this result could not be extended to quasi-geostrophic turbulence which follows a similar phenomenology. A $k^{-3}$ energy spectrum for the ocean currents would then imply a $k^{-1}$ law for the passive scalar; conversely, a $k^{-3}$ spectrum for the passive scalar variance of the type reported by Gower et al. ${ }^{1}$ would imply a much steeper slope $\left(k^{-5}\right)$ for the current kinetic energy.

Such steep slopes have been found in fact in numerical simulations of forced two-dimensional turbulence ${ }^{4}$; the consequent discrepancy with the Kolmo-
gorov-Kraichnan-Leith theory ${ }^{5}$ which predicts a $k^{-3}$ law is still an open question. For instance, the presence of intermittency effects would yield a steepening of the energy spectrum ${ }^{4}$; but still, neither kinetic energy spectra nor temperature variance spectra steeper than $k^{-3}$ have yet been observed experimentally.

The quasi-geostrophic theory of Charney ${ }^{2}$ neglects the influence of upper and lower boundaries; it is therefore likely to be valid in the deep ocean rather than near the surface. Near the surface the dynamics is likely to involve the occurrence of frontal systems, and one should refer to the quasi-geostrophic theory of Blumen ${ }^{6}$ rather than to that of Charney ${ }^{2}$. In Blumen's ${ }^{6}$ theory potential vorticity is assumed to vanish

$$
\psi_{x x}+\psi_{y y}+\psi_{z z} \equiv 0
$$

inside the fluid, and the two invariants are the total energy of the fluid, and the available potential energy at the surface, which both reduce to integrals taken over the surface only:

$$
\begin{aligned}
& \frac{\mathrm{d}}{\mathrm{d} t}\left\langle\psi_{z}^{2}\right\rangle=0 \\
& \frac{\mathrm{d}}{\mathrm{d} t}\left\langle\psi_{z z}^{2}\right\rangle=0
\end{aligned}
$$

The quantity which cascades to smaller scales is the available potential energy at the surface, which therefore exhibits a $k^{-5 / 3}$ law characteristic of frontal systems. If we follow this theory, which looks more appropriate than the first one in the case of surface dynamics, we end up with a $k^{-5 / 3}$ law of the passive scalar variance. This is again quite far from what has been observed for phytoplankton, although again the presence of intermittency could yield a steeper slope.

It seems, therefore, difficult to infer anything conclusive from the measurements reported by Gower et al. ${ }^{1}$ concerning a possible behaviour of phytoplankton as a passive scalar. Also, some caution

\section{Matters Arising}

Matters Arising is meant as a vehicle for comment and discussion about papers that appear in Nature. The originator of a Matters Arising contribution should initially send his manuscript to the author of the original paper and both parties should, wherever possible, agree on what is to be submitted. Neither contribution nor reply (if one is necessary) should be longer than 300 words and the briefest of replies, to the effect that a point is taken, should be considered. should be observed regarding regression estimates for spectral slopes. The concept of an inertial range is only asymptotically valid, and the data used by Gower et al. ${ }^{1}$ may be close to the internal radius of deformation to yield any sensible approximation to the actual behaviour of the inertial range. Apparently a regression calculated on the range $1-10 \mathrm{~km}$ would yield much lower values of the slope.

A secondary point concerns the assumptions in Gower et al. ${ }^{1}$ that radiance upwelling is a linear function of phytoplankton concentration. The only assumption needed to investigate the passive scalar character of phytoplankton is that radiance upwelling is a function of phytoplankton concentration only, because any function of a convected quantity is a convected quantity, exhibiting therefore the same spectral behaviour.

\section{MARCEL LESIEUR}

Institut de Mécanique,

Université de Grenoble,

BP53, Centre de Tri,

38041 Grenoble Cedex, France

\section{ROBERT SADOURNY}

Laboratoire de Météorologie Dynamique, Ecole Normale Supérieure, 24 rue Lhomond, 75005 Paris, France

\footnotetext{
1. Gower, J. F. R., Denman, K. L. \& Holyer, R. J. Nature 288, $157-159(1980)$

2. Charney, J. G. J. atmos. Sci. 8, 1087-1093 (1971)

3. Lesieur, M., Sommeria, J. \& Holloway, G. C. R. hebd. Séanc Acad. Sci., Paris 292, 272-274 (1981).

4. Basdevant, C., Legras, B., Sadourny, R. \& Beland, M. $J$. atmos. Sci. (in the press).

5. Kraichnan, R. H. Phys. Fluids 10, 1417-1423 (1967).
}

6. Blumen, W. J. atmos. Sci, 35, 774-783 (1978).

GOWER ET AL. REPLY-In our recent paper ${ }^{1}$, the -2.92 slope found for the spectrum of near-surface phytoplankton variance is not consistent with theories of a passive scalar in a quasi-geostrophic turbulent fiuid. The reasoning is based on a subsequent paper by Lesieur et al. ${ }^{2}$.

However, we do not feel that a further search for physical explanations for our spectrum is warranted: phytoplankton are not always a conserved passive scalar. Rather, as microscopic plants in the sea, they grow, at times at rates capable of doubling their weight each day; and they die or are eaten, of ten at rates comparable with their growth rates. These time scales are short compared with typical time scales for quasi-geostrophic eddies (10 days). Even in the absence of turbulent motion, biological processes can resuit in similar negative power law spectra ${ }^{3}$. Still it is feasible that areas of abundant phytoplankton may correlate with fluctuations of a dynamic variable, such as upwards 